\title{
Reproductive Allocation Strategy of Two Herbaceous Invasive Plants Across Different Cover Classes
}

\author{
Congyan Wang ${ }^{1,2 *}$, Jiawei Zhou ${ }^{1}$, Jun Liu ${ }^{1}$, Lei Wang ${ }^{1}$, Hongguang Xiao ${ }^{1}$ \\ ${ }^{1}$ Institute of Environment and Ecology, School of the Environment and Safety Engineering, Jiangsu University, \\ Zhenjiang 212013, P. R. China \\ ${ }^{2}$ State Key Laboratory of Soil and Sustainable Agriculture, Institute of Soil Science, Chinese Academy of Sciences, \\ Nanjing, 210008, P. R. China
}

Received: 16 April 2016

Accepted: 15 July 2016

\begin{abstract}
Plant invasion succeeds because of such invader characteristics as fecundity and high-efficiency multiple reproduction modes. The acquisition of individual resources and the benefits of individual fitness are driven by optimum patterns of life history and trade-offs of reproductive allocation (RA) in plants, and variations in RA strategy play an key role in plant adaptation to environmental changes. Thus determining the RA strategy of invasive plants is important for understanding the successful mechanism underlying plant invasion. This study aims to determine the reproductive allocation (RA) strategy of two herbaceous invasive plants (Conyza canadensis and Solidago canadensis) across different cover classes in eastern China. Plant height, maximum branch length, the reproductive branch number, aboveground biomass, the amount of reproductive investment, and the coefficient of RA of the two plants decreased with increasing cover class (although the changes were not pronounced). Thus the two plants may decrease physiological investment on reproductive behavior and reduce RA under competitive conditions because of interspecies competition that progressively decreased and intraspecific competition that gradually increased with increasing cover class. The RA of the two plants may be principally influenced by plant community composition at low cover classes, but by soil $\mathrm{pH}$ at high cover classes. This may be the outcome that soil $\mathrm{pH}$ of the two plants decreased with increasing cover class significantly. Thus, intraspecific competition for resources may play an important role in the RA strategy of the two plants under high cover class.
\end{abstract}

Keywords: invasive plant, cover classes, reproductive allocation strategy, Conyza canadensis, Solidago canadensis

*e-mail: liuyuexue623@163.com 


\section{Introduction}

Invasive plants significantly affect the structure and/ or functions of their surrounding ecosystems [1-3]. Thus, ecologists have recently paid special attention to the successful invasion mechanism of those invaders [1-2] - particularly their reproductive allocation strategy (RA, i.e., the proportion of biomass allocated to reproductive structures) [4]. Some invasive plants successfully invade certain environments because those species feature fecundity and high-efficiency multiple reproduction modes [4-5]. Meanwhile, functional traits determine how plants reproduce and gain resources [6], and such traits affect the success of a species in competition with other species for living resources [7-8]. The acquisition of individual resources and the benefits of individual fitness are driven by optimum patterns of life history as well as trade-offs of RA in plants [9]. Generally, herbaceous plants like to choose $r$ strategies (i.e., increased quantity of offspring at the expense of individual parental investment) rather than $K$ strategies (i.e., reduced quantity of offspring with a corresponding increased parental investment) during their life-history [10-12]. Thus growth and reproduction in plant populations are likely to be optimized only via improved individual resources and fitness, thereby ultimately resulting in the largest number and maximum fitness of future generations [13-15]. Plant reproduction behaviors are closely associated with their acquired living resources $[13-14,16]$. A resource competition exists between growth and reproduction in plants, and the RA strategy of plants controls and balances the dynamics between growth and reproduction [13-17] because living resources in a habitat form one of the most important environmental factors of survival [18]. Meanwhile, the variations in RA strategy play an important role in plant adaptation to environmental changes $[14-15,19]$. Thus, determining the RA strategy of invasive plants is important for understanding the successful mechanism underlying plant invasion.

Gradual succession occurs after successful colonization of new ecosystems by invasive species after those invaders are transported far from their natural habitats [20-21]. Thus, those invaders exert different cover classes in the invaded ecosystems $[2,20,22]$. It was therefore expected that the RA strategy of invasive plants varies in cover classes. Meanwhile, more than two invasive plants generally exist in many natural ecosystems. Unfortunately, studies on the RA strategy of invasive plants mainly focus on single species or differences between invasive and native species. Such studies often ignore the cover classes of invasive plants or do not focus on differences in the RA strategies between different invasive plants (co-occurrence of invasive plants is common in many ecosystems) [2324].

This study was conducted using cross-site comparisons to provide a relatively complete depiction of the RA strategies of two herbaceous invasive plants (Conyza canadensis (L.) Cronq. and Solidago canadensis L.) across different cover classes in nine sampling sites in three provinces in P. R. China. The two plants could be found in the same natural habitat. The two plants are members of the Compositae family and originate from North America [25].

C. canadensis is a herbaceous annual and non-clonal species [25]. It was first recorded in Yantai, Shandong province of China in 1862 [26], and is now widely distributed throughout the 28 provinces and listed as one of the most destructive and widespread invasive species in China [27]. This species thrives in many habitats, including meadows, edges of forests, clearings, orchards, farmland, pond surroundings, stream-banks, and roadsides [28].

$S$. canadensis is a herbaceous perennial and clonal species [25]. S. canadensis was introduced to Shanghai, China, as a horticultural plant in the early 1930s. $S$. canadensis has become naturalized in most of China and recognized as one of the most destructive and widespread invasive species in the country [29-31]. Branch number, reproductive branch number, maximum length of branch, maximum angle of branch, frequency of reproductive investment, the amount of reproductive investment, and the coefficient of RA $\left(\mathrm{C}_{\mathrm{RA}}\right)$ of the two plants are measured to characterize their RA strategy. The results of this study could provide a platform to better underlie the mechanism of successful invasion of the two plants and then lay an important theoretical foundation and practical significance for effective invasion prevention and control.

This study presents the following hypotheses:

1) $\mathrm{C}_{\mathrm{RA}}$ of the two plants decreases with increasing cover class because the RAs of plant populations decrease under competition condition [13, 32].

2) $\mathrm{C}_{\mathrm{RA}}$ of $C$. canadensis is higher than that of $S$. canadensis because annual plants exhibit higher RA than perennial species [33-34], and clonal plants are often characterized by reduced sexual reproduction compared with non-clonal species [35-36], and thus recruitment from seeds is thought to be infrequent [37].

3) The species with higher biomass can display higher $\mathrm{C}_{\mathrm{RA}}$ because they can allocate more biomass into reproductive behaviors when they reach a certain size $[17,19]$.

\section{Materials and Methods}

\section{Site Description}

Samples of the two herbaceous invasive plants $(C$. canadensis and $S$. canadensis) were obtained from nine sampling sites in three provinces in P. R. China, namely, Jiangsu Province - including Nantong $\left(31^{\circ} 97^{\prime} \mathrm{N}\right.$, $\left.120^{\circ} 91^{\prime} \mathrm{E}\right)$, Huai' an $\left(33^{\circ} 51^{\prime} \mathrm{N}, 119^{\circ} 16^{\prime} \mathrm{E}\right)$, and Zhenjiang $\left(32^{\circ} 20^{\prime} \mathrm{N}, 119^{\circ} 52^{\prime} \mathrm{E}\right)$; Zhejiang Province - including Hangzhou $\left(30^{\circ} 21^{\prime} \mathrm{N}, 120^{\circ} 18^{\prime} \mathrm{E}\right)$, Shaoxing $\left(30^{\circ} 10^{\prime} \mathrm{N}\right.$, $\left.120^{\circ} 54^{\prime} \mathrm{E}\right)$, and Jiaxing $\left(30^{\circ} 69^{\prime} \mathrm{N}, 120^{\circ} 80^{\prime} \mathrm{E}\right)$; and Anhui Province - including Hefei $\left(31^{\circ} 86^{\prime} \mathrm{N}, 117^{\circ} 26^{\prime} \mathrm{E}\right)$, Bengbu $\left(32^{\circ} 90^{\prime} \mathrm{N}, 117^{\circ} 45^{\prime} \mathrm{E}\right)$, and Chuzhou $\left(32^{\circ} 31^{\prime} \mathrm{N}, 118^{\circ} 33^{\prime} \mathrm{E}\right)$. The two herbaceous invasive plants were present together in some sampled sites. 


\section{Experimental Design}

From August to September 2013, samples of $C$. canadensis and $S$. canadensis were collected during their reproductive period from the aforementioned sampling sites. The type of plant communities in the sampling site was weed communities. The cover classes of the two plants were evaluated using their coverage (C) in the invaded sites and divided into three levels: low $(0 \%<\mathrm{C} \leq 35 \%, \mathrm{~L})$, moderate $(35 \%<\mathrm{C} \leq 75 \%, \mathrm{M})$, and high $(75 \%<\mathrm{C} \leq 100 \%, \mathrm{H})[2]$. Three plant sample replicates of the same species from the same quadrat $(1 \times 1 \mathrm{~m})$ in the same sampling site were collected randomly. Three rhizospheric soil samples from the same quadrat in the same sampling site of the same species were also collected randomly to measure soil physicochemical properties.

\section{Determining Plant Characteristics and Soil Physicochemical Properties}

Branch and reproductive branch numbers were determined by counting. Plant height, crown diameter, and the maximum length of branch were determined by using a ruler. The maximum angle of branching was detected by using a protractor. Plant biomass was detected by weighing. The belowground-aboveground biomass ratios (B/A) were also computed using the ratio of belowground and aboveground biomass.

The RA strategy of the two plants was assessed using the frequency of reproductive investment, the amount of reproductive investment, and $\mathrm{C}_{\mathrm{RA}}$. The frequency of reproductive investment was computed using the ratio of reproductive branch to branch number, and the amount of reproductive investment was calculated using the biomass of reproductive organs. $\mathrm{C}_{\mathrm{RA}}$ was computed using the ratio of the biomass of reproductive organs to total biomass [38].

Shannon-Wiener diversity $\left(H^{\prime}\right)$ and Pielou evenness $\left(E_{H}\right)$ indices were used to estimate the community structure of the sites on which the two plants were growing. $H^{\prime}$ was determined by the following equation: $H^{\prime}=-\Sigma P_{i} \ln P_{i}$ [39], $P_{\mathrm{i}}$ was calculated as follows: $P_{\mathrm{i}}=n_{\mathrm{i}} / N$, where $n_{\mathrm{i}}$ is the number of individuals of one species and $N$ is the sum of the number of all species in the surveyed area. $E_{H}$ was calculated by the following equation: $E_{H}=H^{\prime} / \ln S$ [40], where $S$ is defined as the number of all species in the surveyed area.

Soil $\mathrm{pH}$ values and moisture were determined using a digital soil acidity-moisture meter (ZD instrument ZD-06, China) [41-42].

\section{Statistical Analysis}

One-way ANOVA was performed to evaluate the differences among various dependent variables between groups followed by multiple comparisons using the Bonferroni correction test at a probability level of 0.01 when appropriate. Unless otherwise stated, $P \leq 0.01$ was considered to indicate statistically significant differences to reduce the occurrence probability of type I error. Patterns between various dependent variables were examined by correlation analysis (using Pearson productmoment correlation coefficient) and principal component analysis (PCA) using SPSS (version 17.0).

\section{Results}

Differences were observed between the morphological characteristics of $C$. canadensis and $S$. canadensis and the soil physicochemical properties across different cover classes (Table 1). In particular, plant diversity in the distribution area of $C$. canadensis and soil $\mathrm{pH}$ at high cover class were significantly lower than those at low and moderate cover classes $(P<0.01)$. Plant evenness in the distribution area of $C$. canadensis at high cover class was significantly lower than that for low cover classes $(P<0.01)$. Crown diameter, maximum length of branch, total biomass, aboveground biomass, belowground biomass, belowground-aboveground biomass ratio, vegetative biomass, amount of reproductive investment, and $\mathrm{C}_{\mathrm{RA}}$ of $C$. canadensis decreased with increasing cover class, although changes were not pronounced $(P>$ $0.01)$. In addition, the height of $C$. canadensis increased as its invasion degree increased, but this change was not significant $(P>0.01)$. The height of $S$. canadensis at high cover class was significantly lower than that at low cover class $(P<0.01)$. By contrast, the soil moisture of $S$. canadensis at high cover class was significantly higher than that at low and moderate cover classes $(P<0.01)$. The belowground-aboveground biomass ratio of $S$. canadensis increased significantly as its invasion degree increased $(P<0.01)$. The maximum length of branch, reproductive branch number, $\mathrm{C}_{\mathrm{RA}}$, and soil $\mathrm{pH}$ values of $S$. canadensis also decreased with increasing invasion degree, but these changes were not significant $(P>0.01)$.

Differences were also observed in the morphological characteristics and the soil physicochemical properties between $C$. canadensis and $S$. canadensis (Table 2). The frequency of reproductive investment and plant diversity in the area of $C$. canadensis were significantly higher than those of $S$. canadensis (Table $2, P<0.01$ ). By contrast, total, belowground, and vegetative biomass, and B/A of C. canadensis were significantly lower than those of $S$. canadensis (Table 2, $P<0.01$ ). $\mathrm{C}_{\mathrm{RA}}$ of $C$. canadensis was higher than that of $S$. canadensis, but the difference was not significant (Table 2, $P>0.01$ ).

The correlation patterns between the morphological characteristics of the two plants, plant diversity, and soil physicochemical properties were observed via correlation analysis (Table 3). In particular, plant height was positively correlated with total, aboveground, and vegetative biomass, the amount of reproductive investment, and soil $\mathrm{pH}(P<0.01)$. Crown diameter was positively correlated with the maximum length of branch, total, aboveground, and vegetative biomass, the amount of reproductive investment, and $\mathrm{C}_{\mathrm{RA}}(P<0.01)$. 


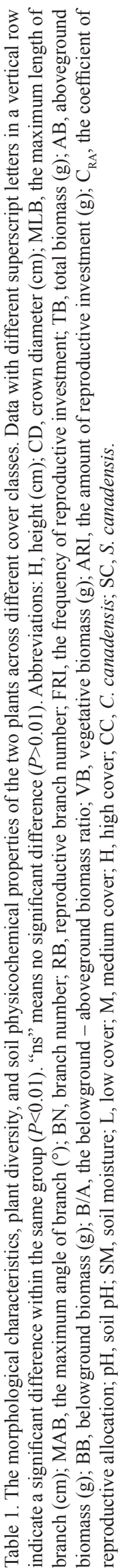

\begin{tabular}{|c|c|c|c|c|c|c|}
\hline$\sum$ & 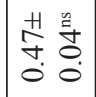 & 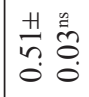 & 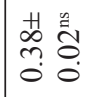 & $\begin{array}{ll}H & 0 \\
\infty & 0 \\
0 & 0 \\
0 & 0\end{array}$ & $\begin{array}{ll}1 & 0 \\
0 & 0 \\
0 & 0 \\
0 & 0 \\
0 & 0\end{array}$ & $\begin{array}{ll}\text { H. } \\
\stackrel{0}{2} \\
0 \\
0\end{array}$ \\
\hline 吉 & 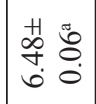 & 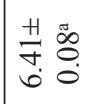 & 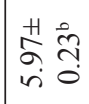 & $\begin{array}{ll}1 & 0 \\
0 & 0 \\
0 & 0 \\
0 & 0 \\
0 & 0\end{array}$ & 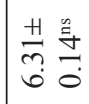 & 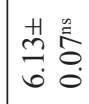 \\
\hline |xt & 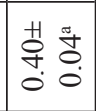 & 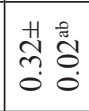 & $\begin{array}{ll}H & 0 \\
& 0 \\
0 & 0 \\
0\end{array}$ & 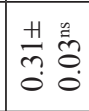 & 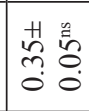 & 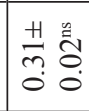 \\
\hline$\Xi$ & 蒙菅 & 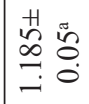 & 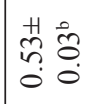 & 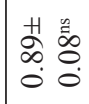 & 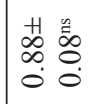 & 䓂产 \\
\hline حُن & 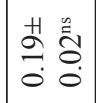 & 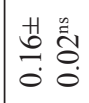 & 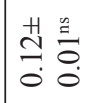 & 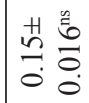 & 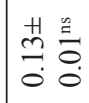 & 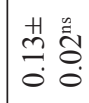 \\
\hline$\overline{\widetilde{z}}$ & 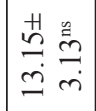 & 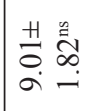 & 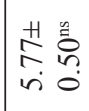 & 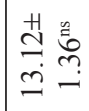 & 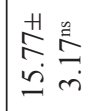 & 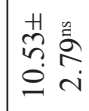 \\
\hline$\stackrel{\infty}{>}$ & 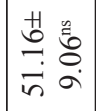 & 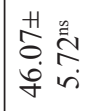 & 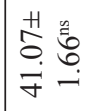 & 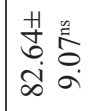 & 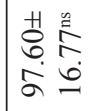 & 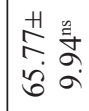 \\
\hline$\overleftrightarrow{\infty}$ & 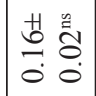 &  & $\begin{array}{l}1 \\
0 \\
0 \\
0 \\
0\end{array}$ & 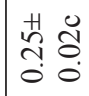 & 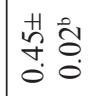 & $\mid$\begin{tabular}{ll}
$H$ & \multicolumn{2}{c}{} \\
$\sigma$ & 0 \\
0 & 0 \\
0 & 0
\end{tabular} \\
\hline$\stackrel{m}{m}$ & 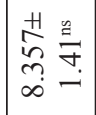 & 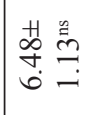 & 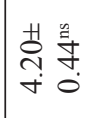 & 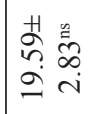 & 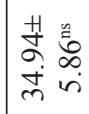 & 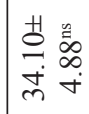 \\
\hline$\frac{m}{4}$ & 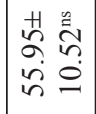 & 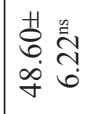 & 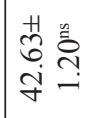 & 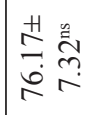 & 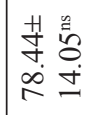 & 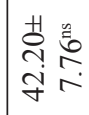 \\
\hline $\mathscr{\vartheta}$ & 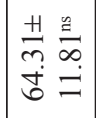 & 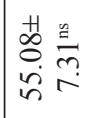 & 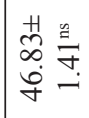 & 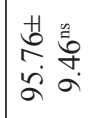 & 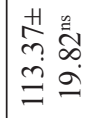 & 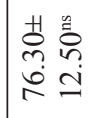 \\
\hline$\frac{\bar{\alpha}}{x}$ & 蓉总 & 荙产 & 蓉言 & 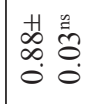 & 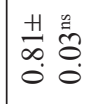 & 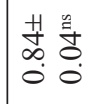 \\
\hline$\frac{p}{\approx}$ & 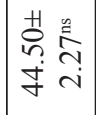 & 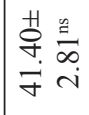 & 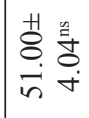 & 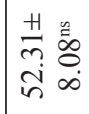 & 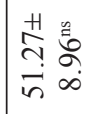 & $\begin{array}{ll}H & 0 \\
0 & 0 \\
0 & 0 \\
\infty & \infty \\
\infty & 0 \\
m & 0\end{array}$ \\
\hline$Z_{m}$ & 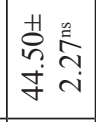 & 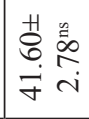 & 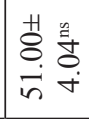 & 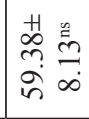 & 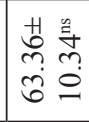 & 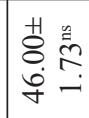 \\
\hline$\stackrel{m}{\Sigma}$ & 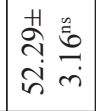 & 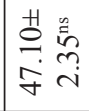 & 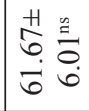 & 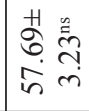 & 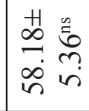 & 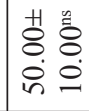 \\
\hline$\stackrel{m}{\xi}$ & 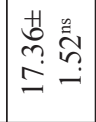 & 营烹 & 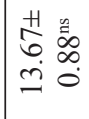 & 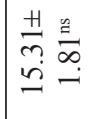 & 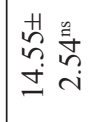 & 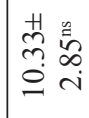 \\
\hline త & 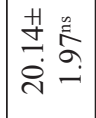 & 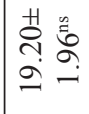 & 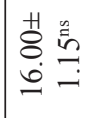 & 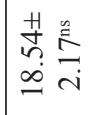 &  & 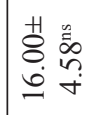 \\
\hline$\Psi$ & 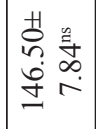 & 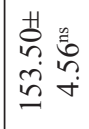 & 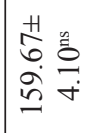 & 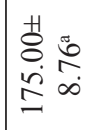 & 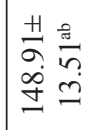 & 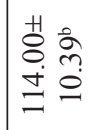 \\
\hline 3 & ـــ & $\Sigma$ & $I$ & - & $\Sigma$ & 工 \\
\hline. $\mathscr{v}$ & \multicolumn{3}{|c|}{ ن } & \multicolumn{3}{|c|}{ v } \\
\hline
\end{tabular}

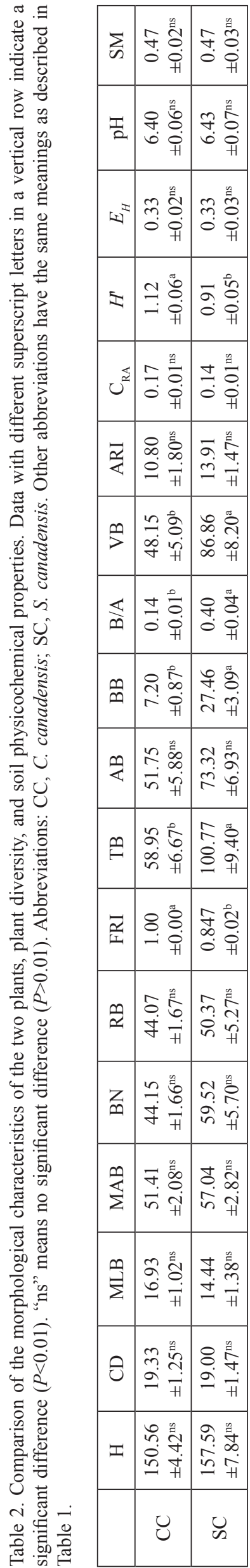


The maximum length of branch was positively correlated with the amount of reproductive investment and $\mathrm{C}_{\mathrm{RA}}$ $(P<0.01)$. The maximum angle of branch was negatively correlated with plant diversity $(P<0.01)$. Branch number was positively correlated with reproductive branch number, total, aboveground, belowground, and vegetative biomass, and the amount of reproductive investment $(P<0.01)$, but negatively correlated with soil moisture $(P<0.01)$. Reproductive branch number was positively correlated with total, aboveground, belowground, and vegetative biomass $(P<0.01)$, but negatively correlated with soil moisture $(P<0.01)$. The frequency of reproductive investment was positively correlated with plant diversity $(P<0.01)$ but negatively correlated with total, aboveground, belowground, and vegetative biomass, and $\mathrm{B} / \mathrm{A}(P<0.01)$. Total, aboveground, belowground, and vegetative biomass were positively correlated with the amount of reproductive investment $(P<0.01)$. The amount of reproductive investment was positively correlated with $\mathrm{C}_{\mathrm{RA}}(P<0.01) . \mathrm{C}_{\mathrm{RA}}$ was also positively correlated with plant diversity $(P<0.01)$.
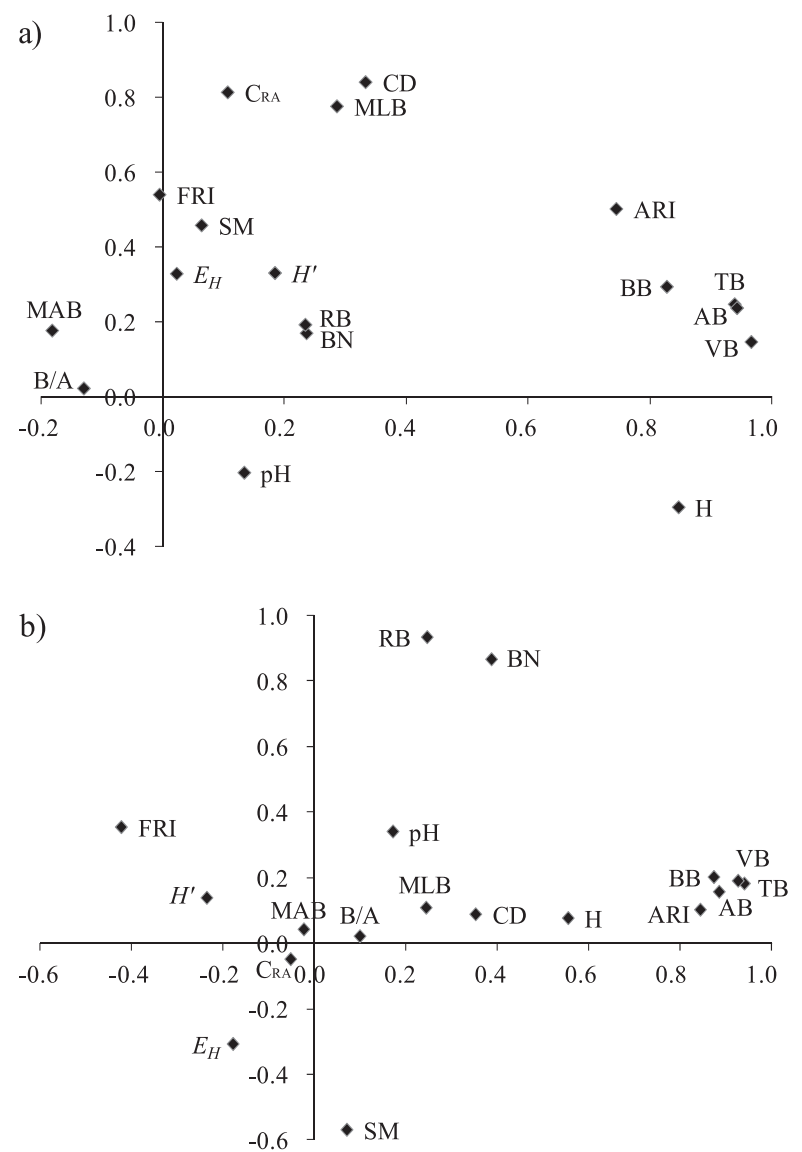

Fig. 1. PCA of the correlation patterns of the morphological characteristics of the two plants, plant diversity, and soil physicochemical properties. The $X$ axis account for $28.19 \%$ and $27.82 \%$ of the total variation in $C$. canadensis a) and $S$. canadensis b), respectively, and the $Y$ axis account for $18.89 \%$ and $13.80 \%$ of the total variation in C. canadensis a) and $S$. canadensis b), respectively. Abbreviations have the same meanings as described in Table 1.
Correlation patterns between the morphological characteristics of the two plants, plant diversity, and soil physicochemical properties were separately examined by PCA (Figs 1 and 2). Many significant correlations were revealed. In particular, strong correlations between $\mathrm{C}_{\mathrm{RA}}$ and crown diameter and the maximum length of
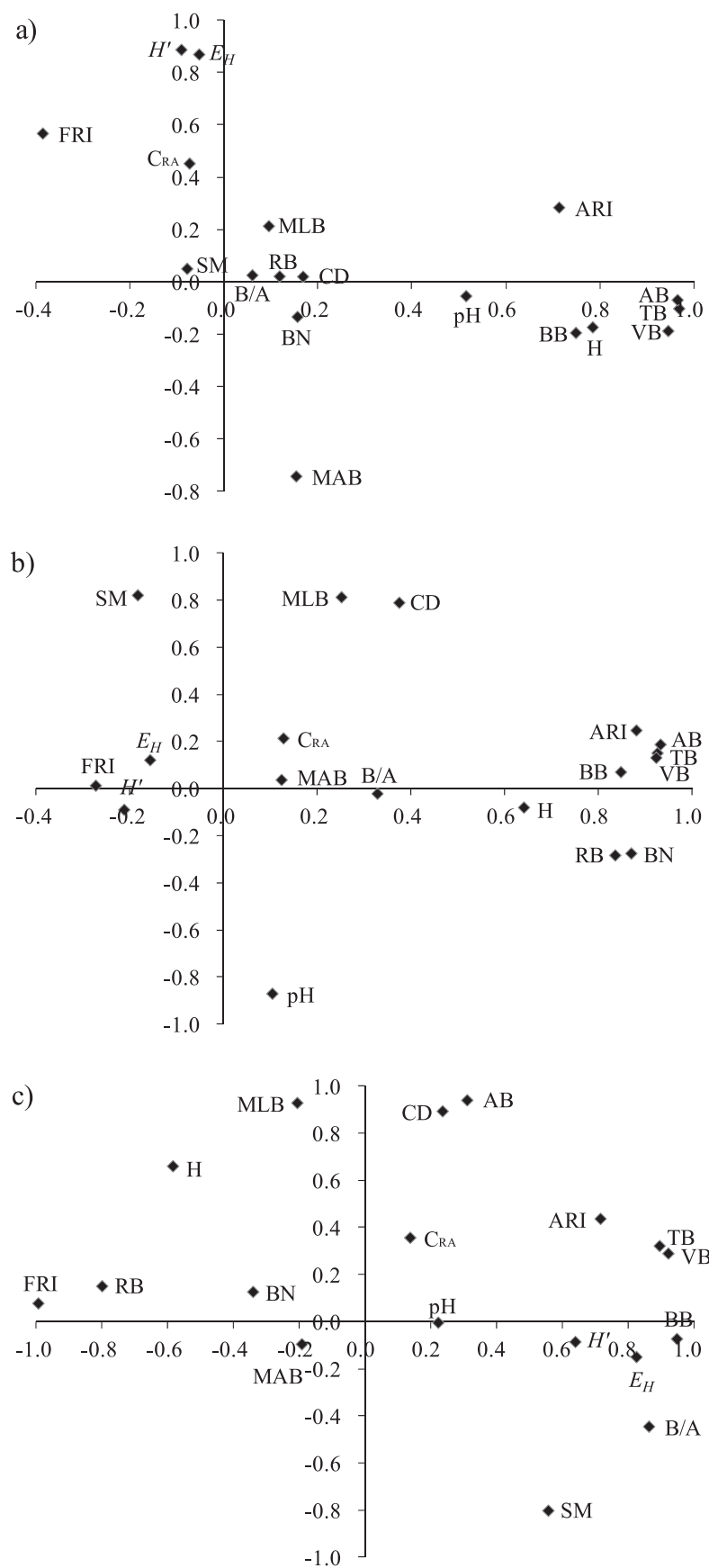

Fig. 2. PCA of the correlation patterns of the morphological characteristics of the two plants, plant diversity, and soil physicochemical properties across different cover classes. The $X$ axis accounts for $27.67 \%, 35.92 \%$, and $42.11 \%$ of the total variation in low a), medium b), and high cover c) of the two plants, respectively, and the $Y$ axis accounts for $16.05 \%, 17.17 \%$, and $24.52 \%$ of the total variation in low a), medium b), and high cover c) of the two plants, respectively. Abbreviations have the same meanings as described in Table 1 . 
branch were observed for C. canadensis (Fig. 1a). Strong correlations between $\mathrm{C}_{\mathrm{RA}}$ and the maximum angle of branch, $\mathrm{B} / \mathrm{A}$, and plant diversity and evenness in the area were observed for S. canadensis (Fig. 1b). Correlations between $\mathrm{C}_{\mathrm{RA}}$ and the maximum length of branch, the frequency of reproductive investment, and plant diversity in the area were strengthened under low cover classes but weakened under moderate and high cover classes (Fig. 2). Correlations between $\mathrm{C}_{\mathrm{RA}}$ and the maximum angle of branch and $\mathrm{B} / \mathrm{A}$ were strengthened under moderate cover classes but were attenuated under low and high cover classes (Fig. 2). Correlations between $\mathrm{C}_{\mathrm{RA}}$ and soil $\mathrm{pH}$ were strengthened under high cover classes, but were attenuated under low and moderate cover classes (Fig. 2).

\section{Discussion}

Invasive plants can display different cover classes in the invaded areas $[2,20]$ due to the gradual succession that occurs after successful colonization of new habitats by invasive species [20-21]. The morphological characteristics of the two plants showed remarkable differences that varied with the cover class in this study. For example, plant height of $C$. canadensis and the belowground-aboveground biomass ratio of $S$. canadensis increased with increasing cover class, although some difference is not significant. Meanwhile, crown diameter, the maximum length of branch, total biomass, aboveground biomass, belowground biomass, the belowground-aboveground biomass ratio, vegetative biomass, the amount of reproductive investment, and $\mathrm{C}_{\mathrm{RA}}$ of $C$. canadensis and plant height, the maximum length of branch and reproductive branch number of $S$. canadensis decreased with increasing cover class, even if some difference is not significant. This may be due to the fact that intraspecific competition may increase at higher cover classes, and changes in plant allocation strategy were consistent with this hypothesis. The results obtained in this study are weakly consistent with our hypothesis. Previous studies also confirmed that plants may decrease physiological investment on reproductive behavior and reduce RA under competitive conditions [13, 32]. Differences in morphological characteristics and RA strategy of the two plants at different cover classes may also result from differences in soil physicochemical properties. Soil $\mathrm{pH}$ values of the two plants decreased with increasing cover classes. Researchers have found that invasive plants induce soil acidification [42-44]. PCA results also revealed that soil $\mathrm{pH}$ were important in determining the RA of the two plants under high cover classes. This result may be the outcome of enhanced soil acidity, which was triggered by the invasion of the two plants. The decreased soil $\mathrm{pH}$ could enhance phosphor (P) solubility in soil [43, 45], and higher $\mathrm{P}$ availability could facilitate further invasion of invasive plants [43-44]. Meanwhile, the results of this study show that $\mathrm{B} / \mathrm{A}$ of the two plants increased with increasing cover class significantly. The increase in resource investment in underground parts may enhance the capability of invasive plants to obtain more resources, particularly water (soil moisture of the two plants increased with increasing cover class, although these changes were not significant) and nutrients (such as P).

PCA results indicated that the influence of plant diversity on $\mathrm{C}_{\mathrm{RA}}$ of the two plants under moderate and high cover class was attenuated. This means that interspecies competition progressively decreased and intraspecific competition gradually increased with increasing cover class. The frequency of reproductive investment on $\mathrm{C}_{\mathrm{RA}}$ of the two plants was attenuated under moderate and high cover classes based on PCA results. This result was consistent with previous studies in which the RAs of plant populations decreased under competitive conditions [13, 32]. Another interesting discovery is that correlations between $\mathrm{C}_{\mathrm{RA}}$ and soil $\mathrm{pH}$ were strengthened under high cover classes, whereas correlations between $\mathrm{C}_{\mathrm{RA}}$ and plant diversity were attenuated under high cover class. Thus the RA of the two plants may be principally influenced by plant community composition at low-cover classes, but was affected by soil $\mathrm{pH}$ at high-cover classes. This implied that intraspecific competition for resources may play an important role in the RA strategy of the two plants under high-cover class. Overall, morphological characteristics and RA strategy of the two plants at different cover classes may be the effects of the interaction of soil $\mathrm{pH}$ and cover class on resource allocation in these two species.

Annual plants may exhibit higher $\mathrm{C}_{\mathrm{RA}}$ than perennial species [33-34] because annual parents must obtain a higher reproductive output and then produce more offspring with higher fitness to offset the loss of future reproductive opportunities [46]. The $\mathrm{C}_{\mathrm{RA}}$ of annual $C$. canadensis is thereby expected to be higher than that of perennial S. canadensis because two important differences between the two species are their pereniality and their ability to use asexual reproduction. $\mathrm{C}_{\mathrm{RA}}$ of $C$. canadensis was higher than that of $S$. canadensis, but the difference was not significant, and this finding was consistent with our hypothesis. Previous results also revealed that clonal plants are often characterized by reduced sexual reproduction compared with non-clonal species [35-36], and recruitment from seeds is considered infrequent [37]. Sexual reproduction, which is important for connecting isolated populations and for colonizing new habitats through seed recruitment and dispersal, contributes much more significantly to quick range expansion of clonal plants than asexual reproduction, thereby enabling rapid colonization of open habitat, formation of densely populated patches, and searching for heterogeneous distributed resources [37, 47]. Thus, the lower RA of clonal plant $S$. canadensis than non-clonal C. canadensis was expected. High RA behavior may play a more important role in the successful invasion of $C$. canadensis. This may also imply that $r$ strategies predominate the lifehistory of $C$. canadensis more than $S$. canadensis [10-12]. Meanwahile, R (ruderal) strategies become dominant in the life-history of $C$. canadensis more than $S$. canadensis [48-49]. The higher RA of C. canadensis may be one of the major reasons behind the distribution of this species 


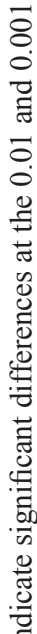

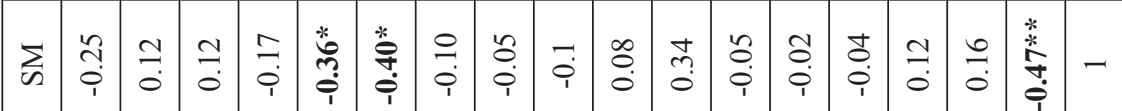

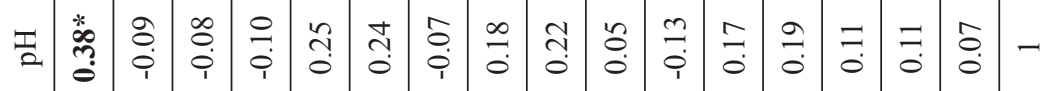

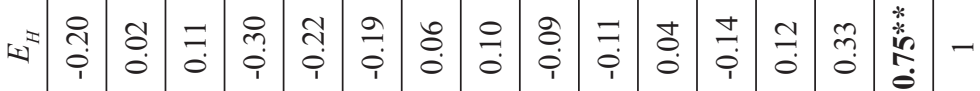

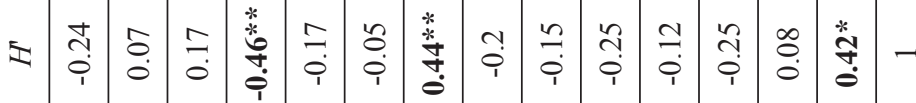

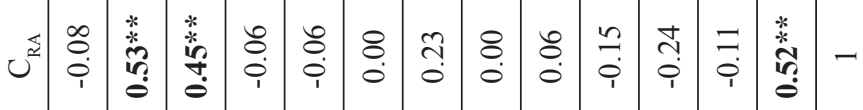

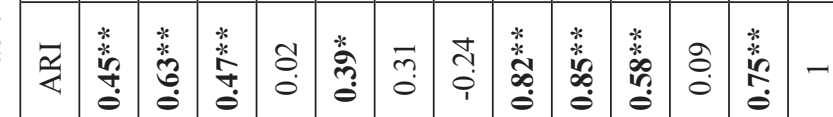

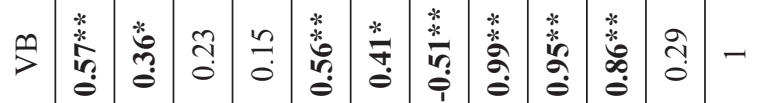

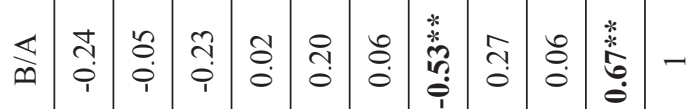

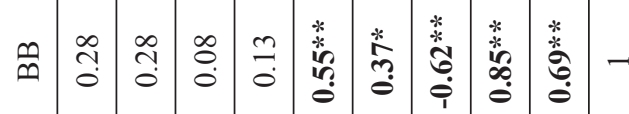

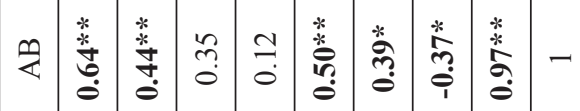

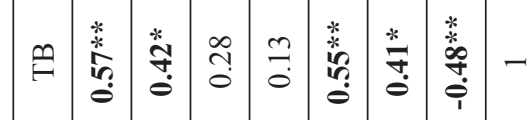

s.

战

흥 흥

范

要

范

늘

둘

窇高

Z

త్

\begin{tabular}{lllll}
$m$ & 0 & 0 & 0 & 0 \\
\hdashline & 0 & 0 & 0 & 0 \\
0 & 0 & 1 & -
\end{tabular}

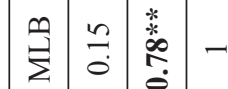

$\theta: \stackrel{0}{0}-$

$\pm-$

这

mi 
in China and why it is renowned as a notorious invasive species. The results also suggest that species specificity exists for RA strategy. This condition may also be due to the difference in the successful ecological strategies that allow plants to obtain maximum fitness advantage and subsequently broaden their habitat niches. Meanwhile, crown diameter and maximum length of branch played important roles in determining $\mathrm{C}_{\mathrm{RA}}$ of $C$. canadensis, and the influence of the maximum angle of branch, B/A, and plant diversity and evenness in the area on $\mathrm{C}_{\mathrm{RA}}$ of $S$. canadensis was strong based on the PCA results. These results suggest that factors that influence the RA vary among species.

Plant species with higher biomass could invest more biomass into reproductive behaviors, thereby showing high $\mathrm{C}_{\mathrm{RA}}[16,19]$. Several researchers have found significant positive correlations between $\mathrm{C}_{\mathrm{RA}}$ and plant size $[16,19]$. In this study, the total, belowground, and vegetative biomass of $S$. canadensis were significantly higher than those of $C$. canadensis, whereas the RA of $S$. canadensis was lower than that of $C$. canadensis. This result may be mainly attributed to the allometric relationship between plant size and $\mathrm{C}_{\mathrm{RA}}$. In other words, the proportions invested into reproductive behavior decrease when individual plants have higher biomass [17]. This phenomenon may be explained by plants investing more biomass in vegetative behavior when they reach a certain size to support their large bodies, i.e., $\mathrm{C}_{\mathrm{RA}}$ decreases with increasing size of individual plants [17]. In this study, we found a negative correlation between total biomass and the frequency of reproductive investment. Unexpectedly, crown diameter and maximum length of branch, rather than plant height or total biomass, were the important characteristics that triggered pronounced effects on RA strategy of the two plants. This result is inconsistent with our hypothesis and with results of previously published studies [19]. Crown diameter was positively correlated with total and aboveground biomass, and amount of reproductive investment and maximum length of branch were positively correlated with the amount of reproductive investment. Thus, crown diameter and the maximum length of branch are important factors that trigger expansion of plant species, thereby enabling them to obtain more living resources - especially light, which is perhaps one of the most important ecological factors that affect plant performance [50].

\section{Conclusions}

This study aimed to address the RA strategy of two herbaceous invasive plants ( $C$. canadensis and $S$. canadensis) across different cover classes in eastern China. Plant height, maximum length of branch, reproductive branch number, aboveground biomass, the amount of reproductive investment, and $\mathrm{C}_{\mathrm{RA}}$ of the two plants decreased with increasing cover class, although the changes were not pronounced. Thus, the two plants may decrease physiological investment on reproduction behavior and reduce RA under competitive conditions because interspecies competition progressively decreased and intraspecific competition gradually increased with increasing cover class. $\mathrm{C}_{\mathrm{RA}}$ of $C$. canadensis was higher than that of $S$. Canadensis, although the difference was not significant. Thus, high RA behavior may play a more important role in the successful invasion of $C$. canadensis. Meanwhile, $r$ strategies predominate the life-history of $C$. canadensis more than $S$. canadensis. In addition, the RA of the two plants may be principally influenced by plant community composition at low cover classes, but by soil $\mathrm{pH}$ at high cover classes. This may be the outcome that soil $\mathrm{pH}$ of the two plants decreased with increasing cover class significantly. Thus, intraspecific competition for resources may play an important role in the RA strategy of the two plants under high cover class.

\section{Acknowledgements}

This study was supported by the National Natural Science Foundation of China (31300343); the Open Science Research Fund of State Key Laboratory of Soil and Sustainable Agriculture, China (Y20160023); and the Research Foundation for Advanced Talents, Jiangsu University (12JDG086). We are very grateful to the anonymous reviewer for insightful and constructive comments that greatly improved this manuscript.

\section{References}

1. POWELL K.I., CHASE J.M., KNIGHT T.M. Invasive plants have scale-dependent effects on diversity by altering speciesarea relationships. Science. 339, 316, 2013.

2. SI C.C., LIU X.Y., WANG C.Y., WANG L., DAI Z.C., QI S.S., DU D.L. Different degrees of plant invasion significantly affect the richness of the soil fungal community. PLoS ONE. 8, e85490, 2013.

3. WANG C.Y., XIAO H.G., ZHAO L.L., LIU J., WANG L., ZHANG F., SHI Y.C., DU D.L. The allelopathic effects of invasive plant Solidago canadensis on seed germination and growth of Lactuca sativa enhanced by different types of acid deposition. Ecotoxicology. 25, 555, 2016.

4. CASTRO S., FERRERO V., COSTA J., SOUSA A.J., CASTRO M., NAVARRO L., LOUREIRO J. Reproductive strategy of the invasive Oxalis pescaprae: distribution patterns of floral morphs, ploidy levels and sexual reproduction. Biol. Invasions. 5, 1863, 2013.

5. MURRAY B.R., PHILLIPS M.L. Investment in seed dispersal structures is linked to invasiveness in exotic plant species of south-eastern Australia. Biol. Invasions. 12, 2265, 2010.

6. SUDING K.N., LAVOREL S., CHAPIN F.S., CORNELISSEN J.H.C., DÍAZ S., GARNIER E., GOLDBERG D., HOOPER D.U., JACKSON S.T., NAVAS M.-L. Scaling environmental change through the communitylevel: a trait-based response-and-effect framework for plants. Global Change Biol. 14, 1125, 2008.

7. CLELAND E.E. Trait divergence and the ecosystem impacts of invading species. New Phytol. 189. 649. 2011. 
8. SCHARFY D., FUNK A., VENTERINK H.O., GUSEWELL S. Invasive forbs differ functionally from native graminoids, but are similar to native forbs. New Phytol. 189, 818, 2011.

9. OBESO J.R. The costs of reproduction in plants. New Phytol. 155, 321, 2002.

10. PIANKA E.R. On r-and K-selection. Am. Nat. 104, 592, 1970.

11. ABRAHAMSON W.G., GADGIL M. Growth form and reproductive effort in goldenrods (Solidago, Compositae). Am. Nat. 107, 651, 1973.

12. REZNICK D., BRYANT M.J., BASHEY F. $r$-and $K$-selection revisited: the role of population regulation in life-history evolution. Ecology. 83, 1509, 2002.

13. NIU K.C., SCHMID B., CHOLER P., DU G.Z. Relationship between reproductive allocation and relative abundance among 32 species of a Tibetan alpine meadow: Effects of fertilization and grazing. PLoS ONE. 7, e35448, 2012.

14. TIAN D.S., PAN Q.M., SIMMONS M., CHAOLU H., DU B.H., BAI Y.F., WANG H., HAN X.G. Hierarchical reproductive allocation and allometry within a perennial bunchgrass after 11 years of nutrient addition. PLoS ONE. 7, e42833, 2012.

15. WANG X.Z., TAUB D.R., JABLONSKI L.M. Reproductive allocation in plants as affected by elevated carbon dioxide and other environmental changes: a synthesis using metaanalysis and graphical vector analysis. Oecologia. 177, 1075, 2015.

16. WEINER J., CAMPBELL L.G., PINO J., ECHARTE L. The allometry of reproduction within plant populations. J. Ecol. 97, 1220, 2009.

17. WEINER J., ROSENMEIER I., MASSONI E.S., VERA J.N., PLAZA E.H., SEBASTIA M.T. Is reproductive allocation in Senecio vulguris plastic. Botany. 87, 475, 2009.

18. REN M.X., ZHANG Q.G. The relative generality of plant invasion mechanisms and predicting future invasive plants. Weed Res. 49, 449, 2009.

19. HAUTIER Y., RANDIN C.F., STOCKLIN J., GUISAN A. Changes in reproductive investment with altitude in an alpine plant. J. Plant Ecol. 2, 125, 2009.

20. THEOHARIDES K.A., DUKES J.S. Plant invasion across space and time, factors affecting nonindigenous species success during four stages of invasion. New Phytol. 176, 256, 2007.

21. WILSON S.D., PINNO B.D. Environmentally-contingent behaviour of invasive plants as drivers or passengers. Oikos. 122, 129, 2013.

22. SEASTEDT T.R., PYŠEK P. Mechanisms of plant invasions of North America and European grasslands. Annu. Rev. Ecol. Syst. 42, 133, 2011.

23. KUEBBING S.E., CLASSEN A.T., SIMBERLOFF D. Two co-occurring invasive woody shrubs alter soil properties and promote subdominant invasive species. J. Appl. Ecol. 51, 124, 2014.

24. KUEBBING S.E., SOUZA L., SANDERS N.J. Effects of co-occurring non-native invasive plant species on old-field succession. Forest Ecol. Manag. 324, 196, 2014.

25. HAO J.H., QIANG S., CHROBOCK T., VAN KLEUNEN M., LIU Q.Q. A test of Baker's law: breeding systems of invasive species of Asteraceae in China. Biol. Invasions. 13, 571, 2011

26. XIE Y., LI Z.Y., GREGG W.P., DIANMO L. Invasive species in China-an overview. Biodivers. Conserv. 10, 1317, 2001.

27. WEBER E., SUN S.Q., LI B. Invasive alien plants in China: diversity and ecological insights. Biol. Invasions. 10, 1411, 2008.

28. MAIN C.L., STECKEL L.E., HAYES R.M., MUELLER T.C. Biotic and abiotic factors influence horseweed emergence. Weed Sci. 54, 1101, 2006.

29. ABHILASHA D., QUINTANA N., VIVANCO J., JOSHI J. Do allelopathic compounds in invasive Solidago canadensis s.1. restrain the native European flora? J. Ecol. 96, 993, 2008.

30. YANG R.Y., YU G.D., TANG J.J., CHEN X. Effects of metal lead on growth and mycorrhizae of an invasive plant species (Solidago canadensis L.). J. Environ. Sci. 20, 739, 2008.

31. ZHAO S.Y., SUN S.G., DAI C., GITURU R.W., CHEN J.M., WANG Q.F. Genetic variation and structure in native and invasive Solidago canadensis populations. Weed Res. 55, 163, 2015.

32. BONSER S.P., AARSSEN L.W. Interpreting reproductive allometry: individual strategies of allocation explain sizedependent reproduction in plant populations. Perspect. Plant Ecol. 11, 31, 2009.

33. MAO W., GINGER A., LI Y.L., ZHANG T.H., ZHAO X.Y., HUANG Y.X. Life history strategy influences biomass allocation in response to limiting nutrients and water in an arid system. Pol. J. Ecol. 60, 381, 2012.

34. MAO W., LI Y.L., CUI D., ZHAO X.Y., ZHANG T.H., LI Y.Q. Biomass allocation response of species with different life history strategies to nitrogen and water addition in sandy grassland in Inner Mongolia. Chin. J. Plant Ecol. 38, 125, 2014. (In Chinese)

35. MANDUJANO M.C., GOLUBOV J., HUENNEKE L.F. Effect of reproductive modes and environmental heterogeneity in the population dynamics of a geographically widespread clonal desert cactus. Popul. Ecol. 49, 141, 2007.

36. SCHLEUNING M., HUAMAN V., MATTHIES D. Flooding and canopy dynamics shape the demography of a clonal Amazon understorey herb. J. Ecol. 96, 1045, 2008.

37. LIU H.Y., LIN Z.S., QI X.Z., ZHANG M.Y., YANG H. The relative importance of sexual and asexual reproduction in the spread of Spartina alterniflora using a spatially explicit individual-based model. Ecol. Res. 29, 905, 2014.

38. SZYMURA M., SZYMURA T.H. Growth, phenology, and biomass allocation of alien Solidago species in central Europe. Plant Species Biol. 30, 245, 2015.

39. SHANNON C.E., WEAVER W. The Mathematical Theory of Communication. University of Illinois Press, Urbana, Illinois, 1, 1949.

40. PIELOU E.C. The measurement of diversity in different types of biological collections. J. Theor. Biol. 13, 131, 1966.

41. XIAO H.G., WANG C.Y., LIU J., WANG L., DU D.L. Insights into the differences in leaf functional traits of heterophyllous Syringa oblata under different light intensities. J. For. Res. 26, 613, 2015.

42. Wang C.Y., Xiao H.G., Liu J., Zhou J.W., Du D.L. Insights into the effects of simulated nitrogen deposition on leaf functional traits of Rhus typhina. Pol. J. Environ. Stud. 25, 1279, 2016.

43. HERR C., CHAPUIS-LARDY L., DASSONVILLE N., VANDERHOEVEN S., MEERTS P. Seasonal effect of the exotic invasive plant Solidago gigantea on soil $\mathrm{pH}$ and $\mathrm{P}$ fractions. J. Plant Nutr. Soil Sci. 170, 729, 2007.

44. CHEN B.M., PENG S.L., NI G.Y. Effects of the invasive plant Mikania micrantha H.B.K. on soil nitrogen availability through allelopathy in South China. Biol. Invasions. 11, 1291, 2009

45. ZHANG G.N., CHEN Z.H., ZHANG A.M., CHEN L.J., WU Z.J. Influence of climate warming and nitrogen deposition on soil phosphorus composition and phosphorus availability in a temperate grassland, China. J. Arid Land. 6, 156, 2014.

46. CHEN X.X., LIANG Y., QI W., SU M., DU G.Z. Studies on reproductive allocation, floral size and its trade-off with 
floral number of annual Gentiana. Acta Prataculturae Sin. 18: 58, 2009 [In Chinese].

47. WILK J.A., KRAMER A.T., ASHLEY M.V. High variation in clonal vs. sexual reproduction in populations of the wild strawberry, Fragaria virginiana (Rosaceae). Ann. Bot. 104, 1413, 2009.

48. GRIME J.P. Evidence for existence of three primary strategies in plants and its relevance to ecological and evolutionary theory. Am. Nat. 111, 1169, 1977.
49. GRIME J.P. Plant Strategies, vegetation processes, and ecosystem properties. Chichester, UK: John Wiley and Sons, 1979.

50. MENG F.Q., CAO R., YANG D.M., NIKLAS K.J., SUN S.C. Trade-offs between light interception and leaf water shedding: a comparison of shade- and sun-adapted species in a subtropical rainforest. Oecologia. 174, 13, 2014. 